\title{
Differences between Proximal versus Distal Intraorbital Optic Nerve Diffusion Tensor Magnetic Resonance Imaging Properties in Glaucoma Patients
}

\author{
Francesca Bolacchi, ${ }^{1}$ Francesco Giuseppe Garaci, ${ }^{1,3}$ Alessio Martucci, ${ }^{2}$ Alessandro Meschini, ${ }^{1}$ \\ Maria Fornari, ${ }^{1}$ Simone Marziali, ${ }^{1}$ Raffaele Mancino, ${ }^{2}$ Ettore Squillaci, ${ }^{1}$ Roberto Floris, ${ }^{1}$ \\ Luciano Cerulli, ${ }^{2}$ Giovanni Simonetti, ${ }^{1}$ and Carlo Nucci ${ }^{2}$
}

Purpose. To analyze in vivo the diffusion tensor magnetic resonance imaging (DT-MRI) properties of the intraorbital optic nerve at two different levels: Proximal to the optic nerve head $(\mathrm{ONH})$ and distal to the $\mathrm{ONH}$ at the level of the orbital apex in glaucoma patients.

Methods. Twenty-four patients with primary open-angle glaucoma were examined. The categorization into early and severe glaucoma was performed by Hodapp's classification. Fifteen healthy individuals served as controls. DT-MRI was performed with a 3T-MR unit.

Results. At early stage mean diffusivity (MD) values were higher at the proximal site with respect to the distal site. On the contrary, a decrease in fractional anisotropy (FA) was observed only relative to patient stage, independent of optic nerve site. Moreover, at early disease stage an increase in overall diffusivities, was evident at the proximal site, whereas at the distal site a decrease of the largest diffusivity and an increase in both the intermediate and smallest diffusivities were observed. FA and MD measured at the proximal site, had, respectively, the highest sensitivity and specificity in discriminating between healthy and glaucomatous eyes.

Conclusions. Our study represents the first attempt to evaluate in vivo fiber integrity changes along the optic nerve with DTMRI. Optic nerve degeneration appears to be a process that affects differently the proximal and the distal segments of the optic nerve. The complementary high sensitivity of FA with the high specificity of MD at the proximal site may provide reliable indexes for the identification of glaucomatous patients at early stages. (Invest Ophthalmol Vis Sci. 2012;53:4191-4196) DOI: $10.1167 /$ iovs.11-9345

From the ${ }^{1}$ Department of Diagnostic Imaging, Molecular Imaging, Interventional Radiology and Radiotherapy and ${ }^{2}$ Department of Exp. Medicine and Surgery, Ophthalmology Unit, University of Rome Tor Vergata, Rome, Italy; and ${ }^{3}$ IRCCS San Raffaele, Rome, Italy.

Submitted for publication December 17, 2011; revised April 16, 2012; accepted April 25, 2012.

Disclosure: F. Bolacchi, None; F.G. Garaci, None; A. Martucci, None; A. Meschini, None; M. Fornari, None; S. Marziali, None; R. Mancino, None; E. Squillaci, None; R. Floris, None; L. Cerulli, None; G. Simonetti, None; C. Nucci, None

Corresponding author: Francesco Giuseppe Garaci, Department of Diagnostic Imaging, Molecular Imaging, Interventional Radiology and Radiotherapy, University of Rome Tor Vergata, Rome, Italy; garaci@gmail.com.

Investigative Ophthalmology \& Visual Science, June 2012, Vol. 53, No. 7

Copyright 2012 The Association for Research in Vision and Ophthalmology, Inc.
Drimary open-angle glaucoma (POAG) is a group of retinal neuropathies associated with increased intraocular pressure (IOP). POAG usually occurs late in life and involves the progressive degeneration of retinal ganglion cells (RGCs) and their axons, which comprise the optic nerve. ${ }^{1,2}$ Morphological alterations of the optic nerve in POAG include loss of axons combined with reactive glial proliferation. ${ }^{3}$ Several scientific evidences are concordant to state that the site of initial injury in glaucoma appears to be the part of the axon that passes through the optic nerve head (ONH). ${ }^{4,5}$ Indeed, structural ONH remodeling has been shown to be an important feature of glaucomatous optic neuropathy. ${ }^{6-16}$ The intraorbital or retrolaminar optic nerve begins at the posterior border of the lamina cribrosa, where the axons become myelinated and surrounded by the pia mater. Recently, intraorbital optic nerve structural alterations have been documented in vivo in glaucoma patients using diffusion tensor magnetic resonance imaging (DT-MRI). ${ }^{15-17}$ DT-MRI measures the three-dimensional properties of water diffusion, and changes in these measurements may reflect changes in tissue microstructure. ${ }^{18}$ Indeed, it has been shown that measurements of water diffusion anisotropy obtained using diffusion tensor imaging (DTI), e.g., fractional anisotropy (FA), are sensitive to axon degeneration. ${ }^{19}$ In particular, the authors, using magnetic resonance diffusion tensor imaging (MR-DTI), have shown that MR-DTI-derived mean diffusivity (MD) and FA values of the intraorbital optic nerve correlate with glaucoma clinical disease stages. ${ }^{15}$ In the present study the authors expand the previous results by evaluating in vivo in glaucoma patients the DT-MRI properties, including the principal diffusivities, of the intraorbital optic nerve at two different levels: Proximal to the $\mathrm{ONH}$, and distal to the $\mathrm{ONH}$ at the level of the orbital apex in glaucoma patients at early and severe glaucoma clinical stages.

\section{Patients and Methods}

From May 2010 to April 2011, two groups of participants were included in this prospective study: A glaucoma group and a control group. The glaucoma group consisted of 24 subjects with diagnoses of POAG: 19 men (mean age 59 years: range 53-71) and five women, (mean age 55 years: range 53-71 years). Glaucomatous eyes were those that exhibited an IOP of $>21 \mathrm{~mm} \mathrm{Hg}$, optic disc cupping, and visual field defects typical of glaucoma. Visual field defects were assessed by means of standard automated perimetry with the 30-2 program of a visual field analyzer (Humphrey Visual Field Analyzer; Carl Zeiss Meditec, Dublin, CA). Only those visual field defects documented in at least two consecutive reliable examinations were considered. Unreliable visual fields were defined as false-negative $>33 \%$, false-positive $>33 \%$, and fixation losses $>20 \%$. Patients with any intraocular disease, intraocular surgery, ocular trauma, or secondary glaucoma were 


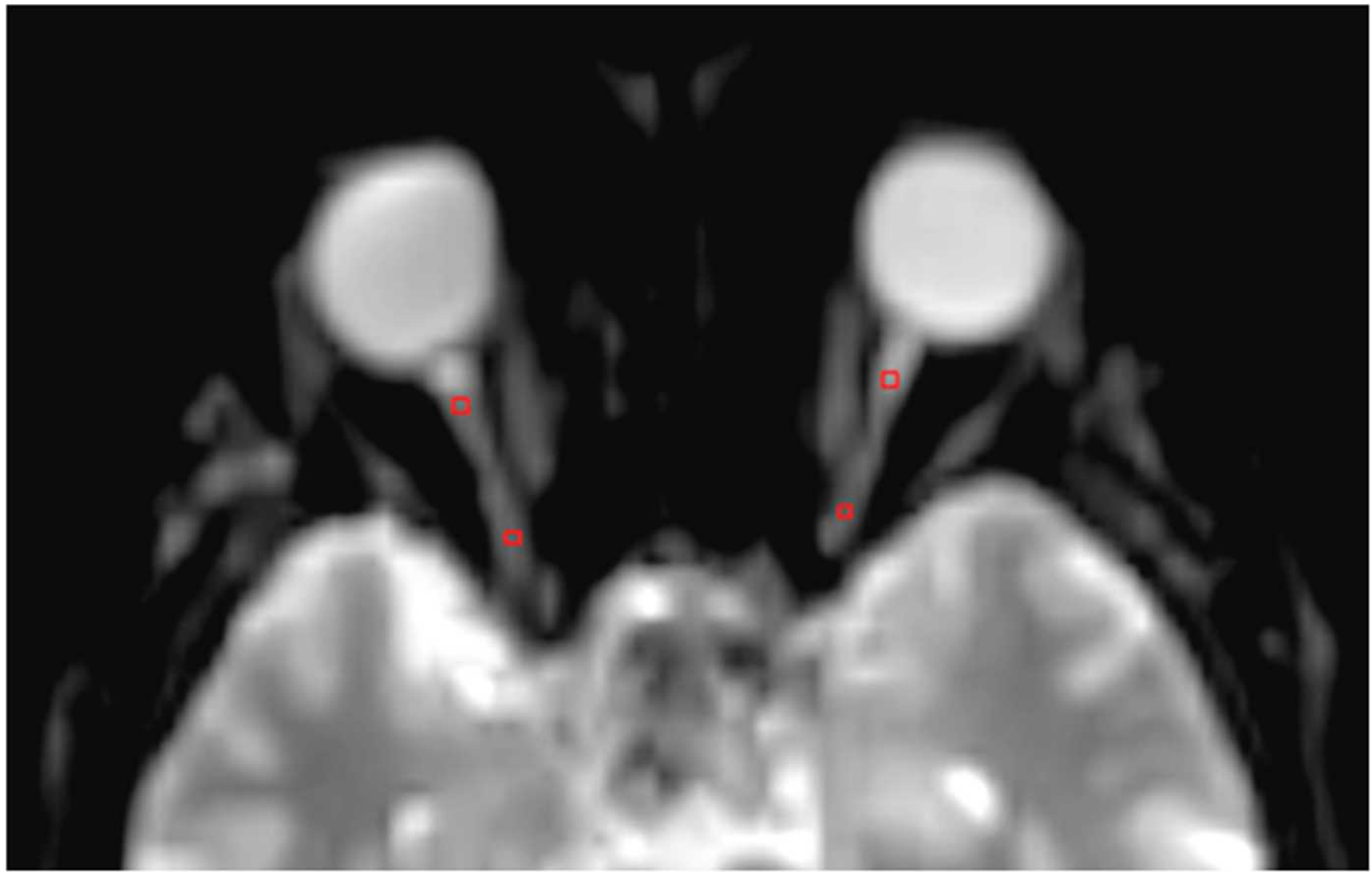

Figure 1. Axial MR image ( $4135 / 59$, b0) shows ROI placements on intraorbital optic nerves. The proximal ROI was positioned $4 \mathrm{~mm}$ posterior to the globe; the distal ROI was positioned $20 \mathrm{~mm}$ posterior to the globe at the level of the orbital apex in 53-year-old man with glaucoma. The boundary of each ROI was carefully defined to achieve the trade-off between consistence of ROI and the influence of partial volume effect.

excluded. The categorization into two subgroups of glaucoma was performed by Hodapp's classification. ${ }^{20}$ Thirty-four eyes were included in the study: 20 eyes with early glaucoma (stage 1 and 2 of the classification) and 14 eyes with severe glaucoma (stage 4 and 5).

Fifteen healthy subjects age-matched and sex-matched to patients were enrolled in the study as controls: 12 men (mean age 62: range, 55-70) and three women (mean age 60, range 52-68). The control eyes had intact neuroretinal rims and no evidence of disc haemorrhage, notching, excavation, or asymmetry of vertical cup-to-disc ratio of more than 0.2. No subject in the control group had a history of intraocular pressure elevation of more than $21 \mathrm{~mm} \mathrm{Hg}$ or ocular trauma. The study was approved by the institutional review board. All participants provided written informed consent; research adhered to the tenets of the Declaration of Helsinki. During the examination the participants were asked to stay with eyes closed in order to minimize ocular bulb movements.

\section{Magnetic Resonance Imaging}

All examinations were performed with a 3-Tesla scanner (Philips Achieva Intera 3T; Philips Healthcare, Eindhoven, The Netherlands). A dedicated 8-element head coil was used for the radiofrequency transmitting signal and for signal reception. Interleaved threedimensional (3D) gradient echo images acquired from the body coil and the sensitivity encoding (SENSE) coil array (acquisition matrix $=$ $482, \alpha=7^{\circ}$, repetition time $(\mathrm{TR})=8 \mathrm{~ms}$, echo time $\left.(\mathrm{TE})=1.6 \mathrm{~ms}\right)$ served as references for sensitivity calibration. For MR-DTI, the investigators used a SENSE-single shot (ssh) SE-echo planar imaging (EPI) sequence (field of view $[\mathrm{FOV}]=250 \times 250$, thickness/gap $=2 / 0$, $\mathrm{TR}=41355 \mathrm{~ms}, \mathrm{TE}=59 \mathrm{~ms}$, SENSE reduction factor $\mathrm{R}=2.4$, partial Fourier encoding $=60 \%$ ) with a maximum b value of $800 \mathrm{~s} / \mathrm{mm}^{2}$ along
32 directions. A baseline image with no diffusion weighting $(b=0)$ was also acquired. Matrices of $256 \times 256$ voxels with an in-plane resolution of $0.8 \times 0.8 \mathrm{~mm}$ were used for diffusion tensor calculation. The total scanning time, including setup and anatomical scans, was kept below 15 minutes.

\section{Data Processing}

Data were processed with an automatic customized script on the magnetic resonance (MR) console (Philips AchievaIntera 3T; Philips Healthcare, Eindhoven, The Netherlands) in consensus by two radiologists (F.G.G. and F.B.) experienced with DT-MRI and blinded to the patient's clinical data. From the DTI data, the authors computed the $\mathrm{MD}, \mathrm{FA}$, and the principal diffusivities $(\lambda 1, \lambda 2$, and $\lambda 3$, in decreasing order of magnitude). Three regions of interest (ROIs) of approximately $5 \mathrm{~mm}^{2}$ (range 4-6) were manually drawn over the reference SE-EPI images $(b=0)$ at the level of the intraorbital optic nerves $4 \mathrm{~mm}$ and 20 $\mathrm{mm}$ posterior to the globe (Fig. 1). The ROIs were electronically superimposed on the diffusion tensor (DT) maps. Data from the proximal and distal ROIs were analyzed separately. To avoid the introduction of bias by partial volume effect, careful note was taken of the signal characteristics of each voxel within the ROI when transferred to the DT maps. Any ROI which contained one or more "empty" voxels, where the DT-MRI calculation failed was excluded. Also, DT images that revealed a contamination of the anterior segment by the posterior aspect of the globe were excluded from the analysis.

\section{Statistical Analysis}

Interexamination reproducibility was assessed by performing the ROI analysis on three different occasions in 10 control subjects. Interex- 


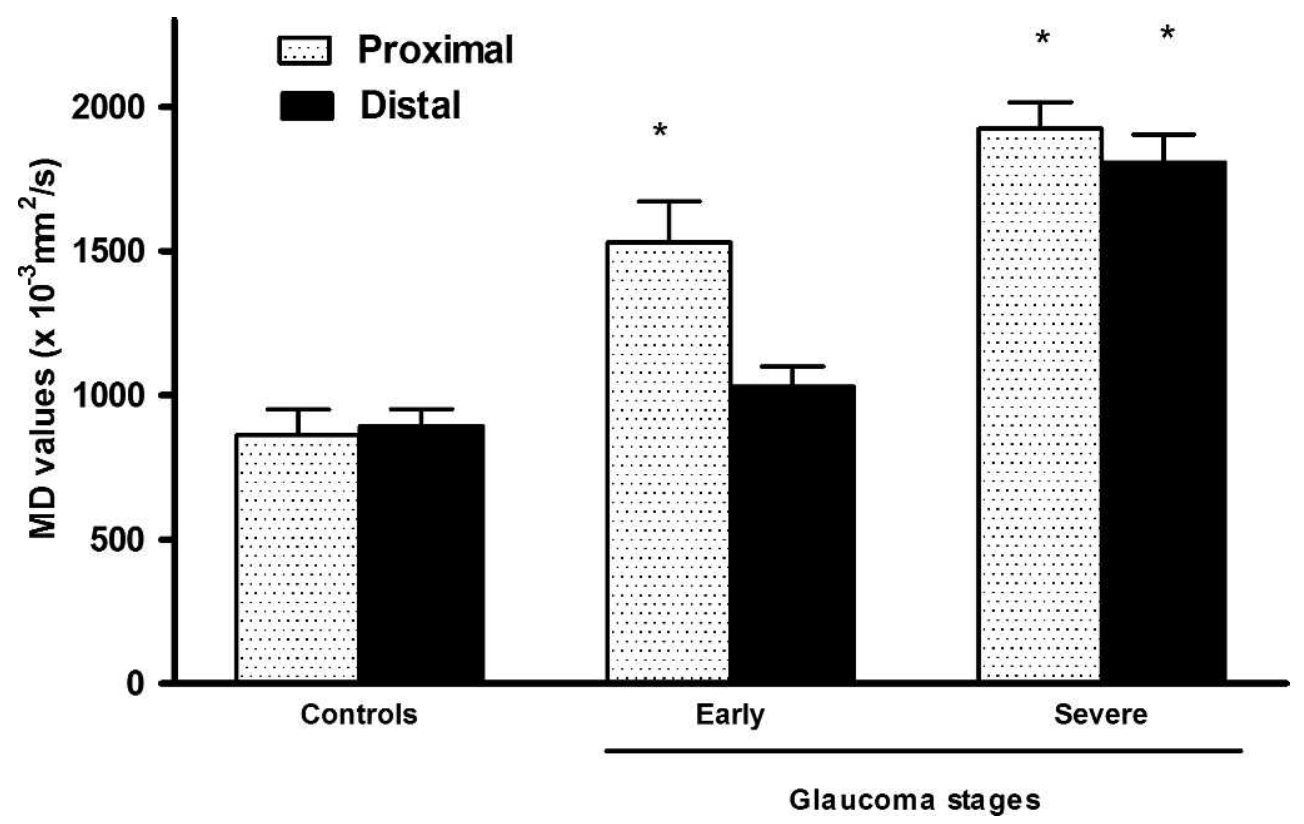

Figure 2. Evaluation of MD values. Measurements of the intraorbital optic nerve MD were taken proximal ( $4 \mathrm{~mm}$ ) and distal (20 mm) to the ocular globe. ${ }^{*} P<0.05$.

amination reproducibility of MD and FA was obtained by measuring the interexamination coefficients of variation (CV). Patients' DTI data were compared to controls using the Mann-Whitney $U$ test. A two-way analysis of variance (ANOVA) was used to assess the effects of glaucoma severity (early and severe), the optic nerve sites ("proximal," and "distal") and their interaction respectively on the DTI parameters. The receiver operating characteristic (ROC) curves analysis was performed to evaluate the diagnostic value of $\mathrm{FA}$ and $\mathrm{MD}$ in discriminating between healthy eyes and glaucomatous eyes. Optimal cut-off values were defined maximizing the sum of sensitivity and specificity. A $P$ value less than 0.05 was considered significant. Data were analyzed using biostatistics curve fitting software (GraphPad Prism version 4.7; GraphPad Software, San Diego, CA) and ROC curve analysis software (MedCalc version 11.3.3.0; MedCalc Software, Mariakerkbe, Belgium).

\section{RESULTS}

Review of the acquired DT images revealed that the most anterior segment of the optic nerve was contaminated by the posterior aspect of the globe in six eyes of four control subjects and in nine eyes of eight patients, which were excluded from the study. Also, signal characteristics of the voxels within the ROI contained one or more "empty" voxels in three eyes of two patients. Thus, 24 control eyes, 12 eyes with early glaucoma, and 10 eyes with severe glaucoma were finally included in the study.

ROIs Analysis Reproducibility. MD mean interexamination coefficient variations were 4.20 and 4.50 , respectively, at the level of the proximal and distal segments. FA mean interexamination coefficient variations were 3.45 and 3.63 as measured respectively at the level of the proximal and distal segments of the intraorbital optic nerve.

MD Analysis. In control subjects no statistically significant difference was observed between the MD values measured in the proximal and distal segments $\left(860 \pm 150 \times 10^{-3}\right.$ vs. $900 \pm$ $\left.170 \times 10^{-3}, P>0.05\right)$. In glaucoma patients at early disease stage the proximal portion of the intraorbital optic nerve showed a statistically significant alteration of the MD values, $\left(860 \pm 150 \times 10^{-3}\right.$ vs. $\left.1550 \pm 180 \times 10^{-3}, P=0.0035\right)$ whereas the distal portion showed a mild but not statistically significant increase with respect to control subjects $(900 \pm$ $270 \times 10^{-3}$ vs. $1080 \pm 152 \times 10^{-3}, P=0.062$ ). In contrast, at severe stages both the proximal and the distal portion of the optic nerve showed altered MD values with respect to control subjects $\left(860 \pm 150 \times 10^{-3}\right.$ vs. $1900 \pm 220 \times 10^{-3}, P=0.003$ and $900 \pm 270 \times 10^{-3}$ vs. $1850 \pm 280 \times 10^{-3}, P=0.002, P=$ 0.062) (Fig. 2). The two-way ANOVA analysis showed a significant difference of the MD values both in relation to the optical segment $(P=0.011)$ and to disease severity $(P<$ $0.0001)$. Moreover, a significant interaction existed between the two main effects of disease severity and optic nerve segment $(P=0.018)$ in affecting MD values. These data indicate that the MD was significantly larger at the proximal site with respect to distal site in patients with early glaucoma.

FA Analysis. In control subjects, no statistically significant difference was observed between the FA values measured in the proximal and distal segments $\left(515 \pm 50 \times 10^{-3}\right.$ vs. $562 \pm$ $60 \times 10^{-3}, P=0.72$ ). In glaucoma patients at early disease stage, the proximal and the distal portions showed a statistically significant decrease of FA with respect to controls $\left(515 \pm 50 \times 10^{-3}\right.$ vs. $390 \pm 43 \times 10^{-3}, P=0.011$ and $562 \pm$ $60 \times 10^{-3}$ vs. $\left.400 \pm 53 \times 10^{-3}, P=0.025\right)$. At severe disease stage, the proximal and the distal portions showed a statistically significant decrease of FA with respect to controls also $\left(515 \pm 50 \times 10^{-3}\right.$ vs. $260 \pm 34 \times 10^{-3}, P=0.001$ and 562 $\pm 60 \times 10^{-3}$ vs. $290 \pm 58 \times 10^{-3}, P=0.001$ ) (Fig. 3). The twoway ANOVA analysis showed no significant difference between the FA values at the proximal and distal locations $(P=0.070)$. Also no significant interaction existed between the two main effects of disease severity and optic nerve segment $(P=0.411)$. However, a significant difference in FA values was observed relative to patient stage, independent of optic nerve site $(P<$ 0.001).

Accuracy of MD and FA in Discriminating between Healthy and Glaucomatous Eyes. Figure 4 shows the diagnostic accuracies in discriminating healthy from glaucomatous eyes as assessed by area under curve-receiver operating curve (AUC-ROC) analysis of the MD and FA values, measured both at the proximal and distal sites. No differences were 


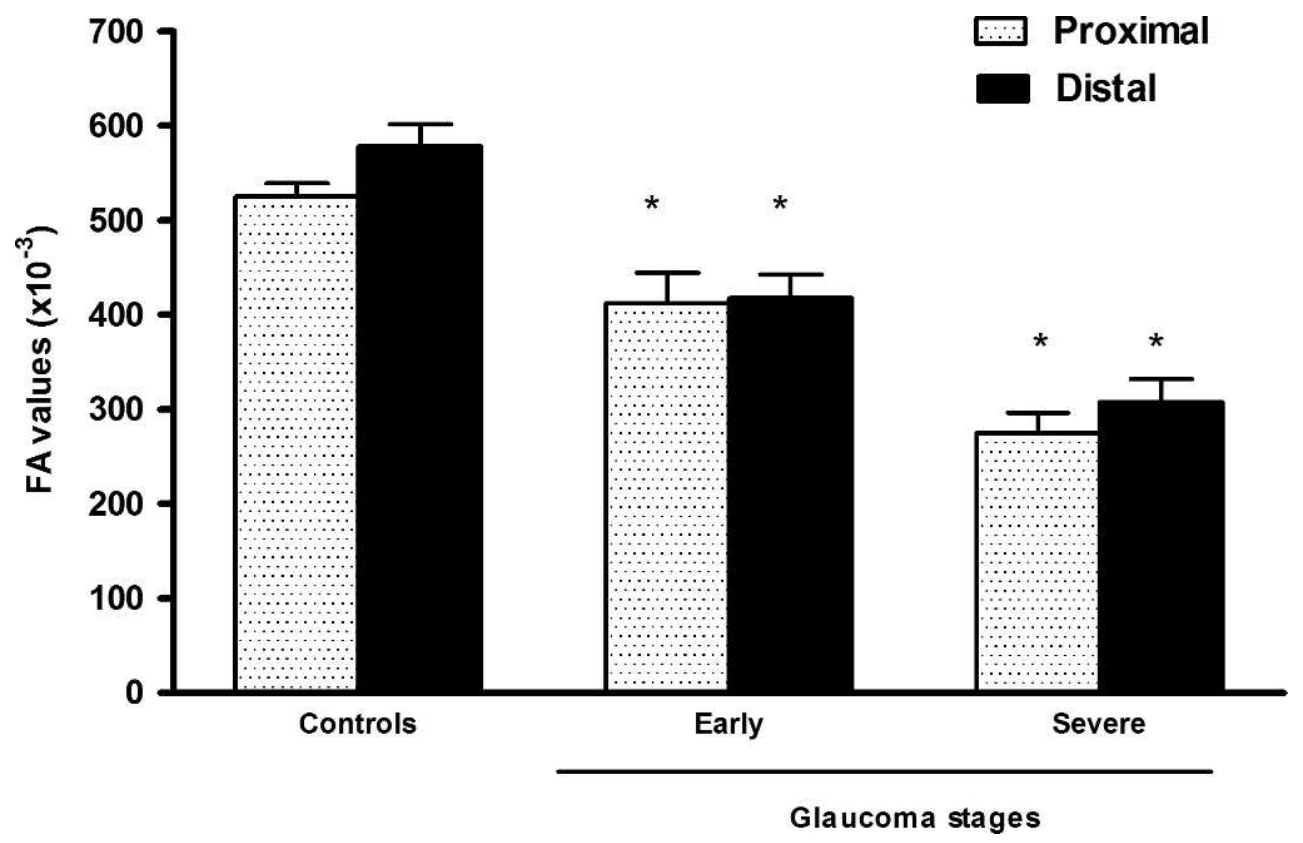

Figure 3. Evaluation of FA values. Measurements of the intraorbital optic nerve FA were taken proximal ( $4 \mathrm{~mm}$ ) and distal (20 $\mathrm{mm})$ to the ocular globe. ${ }^{*} P<0.05$.

observed between the diagnostic accuracy of FA measured at the proximal site and at the distal site $(P=0.07,95 \%$ confidence interval (CI) -0.00416 to 0.134 ). On the contrary, a significant difference was observed between the MD measured at the proximal site and at the distal site $(P=0.02,95 \% \mathrm{CI}$ 0.044 to 0.53 ). FA, irrespective of optic nerve site, and MD measured at the proximal site showed, respectively, the highest sensitivity and the highest specificity: At a cut-off of $\leq 450$, FA showed a specificity of $72 \%$ and a sensitivity of $93 \%$; at a cut-off of $\geq 1400$, MD measured at proximal site showed a specificity of $100 \%$ and a sensitivity of $64 \%$.

Principal Diffusivities Analysis. The analysis of the principal diffusivities at early disease stage showed a statisti-

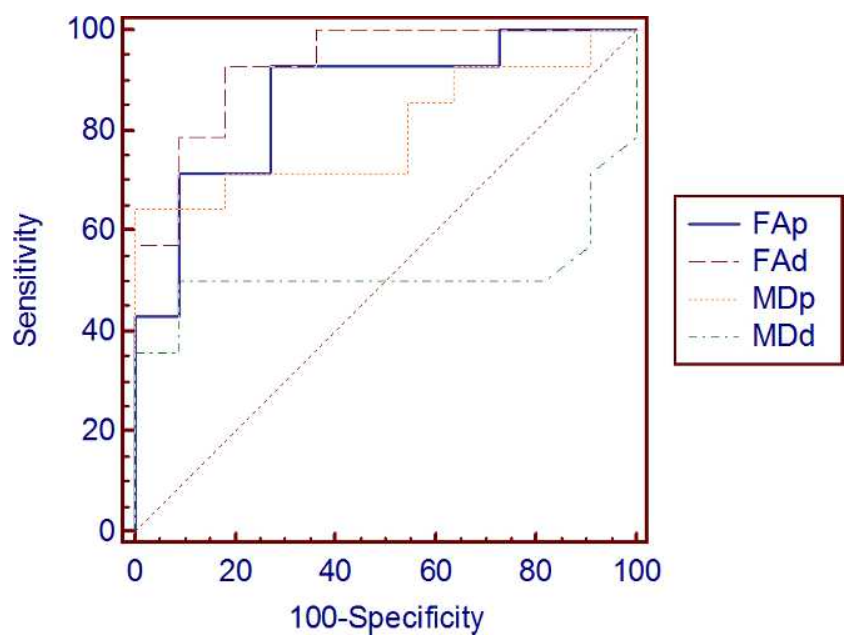

Figure 4. The ROC curves analysis was performed to evaluate the diagnostic value of FA and MD in discriminating between healthy eyes and glaucomatous eyes. The areas under the ROC curves (AUC), of FA measured at the proximal site (FAp), of FA measured at the distal site (FAd), of MD measured at the proximal site (MDp), and of MD measured at the distal site (MDd) were 0.86, 0.92, 0.80, and 0.73, respectively. cally significant increase in both the largest diffusivity $(\lambda 1)$ and in the intermediate and smallest diffusivities ( $\lambda 2$ and $\lambda 3)$ at the level of the proximal segment, whereas at the level of the distal segment, a statistically significant decrease of $\lambda 1$ associated with an increase in $\lambda 2$ and $\lambda 3$ was observed. At severe disease stage a statistically significant increase in overall diffusivities was observed at both the proximal and distal sites (Table).

\section{Discussion}

In the present study the authors expanded their previous results $^{17}$ by analyzing the $\mathrm{MD}$, the $\mathrm{FA}$, and the principal diffusivities of the intraorbital optic nerve at two different levels: Proximal to the $\mathrm{ONH}$ and distal to the $\mathrm{ONH}$ at the level of the orbital apex in glaucoma patients at early and severe glaucoma clinical stages. This study's data indicate that disease severity and optic nerve site interact in affecting MD. In particular, at early disease stage a significant increase in MD values was observed in the proximal segment with respect to the distal site, whereas a significant decrease in FA values was observed only relative to patient stage, independent of optic nerve site. Moreover, at early disease stage an increase in all the principal diffusivities was evident in the proximal segment, whereas at the distal site a decrease of the largest principal diffusivity, $\lambda 1$, and an increase of the intermediate and smallest diffusivities, $\lambda 2$ and $\lambda 3$, were observed. The AUC-ROC analysis showed that FA, irrespective of optical nerve site and MD measured at the proximal site, had, respectively, the highest sensitivity and specificity in discriminating between healthy and glaucomatous eyes.

Several scientific evidences are concordant to state that the site of initial injury in glaucoma appears to be the part of the axon that passes through the ONH. ${ }^{4,5}$ It has been suggested that the structural characteristics of the extra cellular matrix (ECM) are altered by changes in IOP and that these alterations are likely to affect the development of glaucomatous optic nerve damage. ${ }^{6-16}$ The primate $\mathrm{ONH}$ can be considered in four levels. From anterior to posterior, these layers are the nerve fiber layer, the lamina choroidalis, the lamina cribrosa, and the 
TABLE. Principal Diffusivities (Eigenvalues)

\begin{tabular}{|c|c|c|c|c|c|}
\hline \multirow[t]{2}{*}{ Eigenvalue $\left(\mathrm{mm}^{2} \mathbf{s}^{-1}\right)$} & \multirow[b]{3}{*}{ Control } & \multicolumn{4}{|c|}{ Proximal } \\
\hline & & \multicolumn{4}{|c|}{ Glaucoma } \\
\hline & & Early & $\boldsymbol{P}^{*}$ & Severe & $\boldsymbol{P}^{* *}$ \\
\hline$\lambda 1$ & $1.38 \pm 0.13$ & $1.82 \pm 0.14$ & 0.032 & $1.95 \pm 0.59$ & 0.004 \\
\hline$\lambda 2$ & $0.53 \pm 0.13$ & $0.84 \pm 0.22$ & 0.025 & $1.56 \pm 0.32$ & $<0.001$ \\
\hline \multirow[t]{3}{*}{$\lambda 3$} & $0.21 \pm 0.07$ & $0.44 \pm 0.23$ & 0.024 & $1.26 \pm 0.42$ & $<0.001$ \\
\hline & & \multicolumn{4}{|c|}{$\begin{array}{l}\text { Distal } \\
\text { Glaucoma }\end{array}$} \\
\hline & Control & Early & $\boldsymbol{P}^{*}$ & Severe & $\boldsymbol{P}^{* *}$ \\
\hline$\lambda 1$ & $1.42 \pm 0.22$ & $1.14 \pm 0.20$ & 0.033 & $2.04 \pm 0.61$ & 0.003 \\
\hline$\lambda 2$ & $0.48 \pm 0.16$ & $0.84 \pm 0.24$ & 0.031 & $1.74 \pm 0.72$ & $<0.001$ \\
\hline$\lambda 3$ & $0.18 \pm 0.11$ & $0.52 \pm 0.18$ & 0.014 & $1.41 \pm 0.59$ & $<0.001$ \\
\hline
\end{tabular}

Results are reported for the proximal and distal segments of the intraorbital optic nerve in control subjects and in glaucoma patients at early and severe disease stages. Data are expressed as means \pm SD. $P<0.05$ was considered statistically significant.

* Early glaucoma patients versus controls.

** Severe glaucoma patients versus controls.

retrobulbar (retrolaminar) optic nerve. ${ }^{21}$ It is generally assumed that the axonal insult in glaucomatous optic neuropathy involves the scleral lamina cribrosa, a tissue presumed to be easily affected by elevated IOP. However, descriptions of morphological changes in the retrolaminar myelinated region of glaucomatous optic nerves have also been reported. Furuyoshi et al. ${ }^{7}$ investigated the retrolaminar part of the optic nerve in experimentally induced chronic highpressure glaucoma in monkey eyes. The authors reported that in the retrolaminar region axons are destroyed and the space is filled by astrocytes and collapsed connective tissue. Pena et al. ${ }^{6}$ suggested that in the experimental glaucoma eyes, the retrolaminar septa were recruited into the 3D load-bearing structure of the lamina. Hernandez ${ }^{8}$ described a thick dense collagenous matrix separating the nerve bundles at the posterior laminar boundary (where the axons become myelinated) that was not present in normal eyes. There is also evidence suggesting that chronic remodeling of the lamina results in a progressive posterior migration of the laminar insertion into the scleral canal wall, which eventually results in the posterior lamina inserting into the pia mater. ${ }^{11-13}$ Also, data on glaucoma animal models based on dissected $\mathrm{ONH}$ including portions of both the unmyelinated and the initial myelinated segment of the intraorbital (retrolaminar) optic nerve have shown that pressure-induced injury may result in dramatically altered gene expression. ${ }^{14}$ In particular, these authors have found that the mRNA for the water channel protein aquaporin4 (the principle water channel protein in astrocytes, that is associated with astrocytic endfeet and is implicated in neural edema) was significantly decreased in glaucomatous optic nerve. The portion of the retrobulbar axon proximal to the $\mathrm{ONH}$ examined in the present study is proximal to the retrolaminar myelinated neural tissue that is part of the $\mathrm{ONH}$, and shares with the latter the same histopathological features. Thus, the authors speculate that at early disease stages the increase in the MD and the decrease in the FA accompanied by an increase in the principal diffusivities observed in the optic nerve segment proximal to the $\mathrm{ONH}$ may reflect the axonal distress due to the above mentioned structural and functional changes. In particular, the authors speculate that the downregulation of aquaporin- 4 could possibly be responsible for the increase in overall diffusivities in the proximal segment at early stages. Aquaporin- 4 immunolocalizes to the astrocytic endfeet that abut brain microvessels and subarachnoidal spaces and is also expressed by activated microglia. Aquaporins function to maintain neural volume and water homeostasis. The astrocytic endfeet probably play a key role in the transfer of metabolites and water between the axons and the vasculature and subarachnoid space. Indeed, data from electron microscopic studies of optic nerve heads exposed to elevated IOP found vacuoles or swellings associated with $\mathrm{ONH}$ astrocytic endfeet and identified these endfeet as sites of early injury. ${ }^{14}$

On the contrary, in the distal segment the decrease in FA was accompanied by a decrease of the largest principal diffusivity, $\lambda 1$, and an increase of the intermediate and smallest principal diffusivities, $\lambda 2$ and $\lambda 3$. The reduction in FA, accompanied by decreased diffusivity parallel to the fibers $(\lambda 1)$, increased diffusivity perpendicular to them $(\lambda 2-\lambda 3)$ found in distal segment, and with a mild but not significant increase in MD is consistent with the presence of isotropic structures in the distal segment of the intraorbital optic nerve. This can be due to the presence of gliosis, which has been extensively documented in optic nerve degeneration. Indeed, in accordance with Pierpaoli et al., ${ }^{22}$ the authors speculate that fiber loss per se, without an increase in isotropic structures, would be insufficient to produce a decrease in diffusion anisotropy. In fact, if the space occupied by degenerated fibers is simply replaced by neighboring fibers that are still intact the authors expect a reduced volume of anisotropic tracts but not a reduction of the measured diffusion anisotropy. ${ }^{22}$ Moreover, the diffusion properties of the distal segment observed at early stages in the present study are consistent with the MR-DTI "signatures" of Wallerian degeneration evidenced by Pierpaoli et $\mathrm{al}^{22}$

The AUC-ROC analysis indicated that FA values, irrespective of optic nerve site, and the MD values measured at the proximal site demonstrated, respectively, the better sensitivity and specificity to microstructural optic nerve changes. It has been reported that, although FA is a sensitive marker of the tissue microstructural organization it is not a specific marker of disease or myelination. ${ }^{23,24}$ Indeed, it has been suggested that the availability of the regional MD values in addition to scaleindependent anisotropy would increase the diagnosis and the pathologic specificity of the diffusion tensor MR imaging metrics. $^{23}$ Thus, the complementary sensitivity of FA with the specificity of MD evidenced in our study may eventually open up a whole set of exciting new possibilities in the detection of early stage glaucoma in low-pressure open-angle patients before field defects are noticed.

Data from the present study also indicate that at severe disease stages the proximal and the distal segments of the optic nerve are both equally altered with regard to the MD, to the FA values, and to the overall diffusivities. This confirms that 
axonal degeneration, if not treated, is an irreversible process with progressive overall fiber loss.

There are, however, particular challenges associated with in vivo imaging of the optic nerve with magnetic resonance imaging (MRI), and especially with DT-MRI. ${ }^{25}$ The optic nerves are small, mobile structures surrounded by cerebral spinal fluid (CSF) and orbital fat. In particular, ROI-based diffusion tensor imaging studies, such as the present study, include partial volume contamination with adjacent tissues due to the selection of size and location of ROIs. ${ }^{26}$ Indeed, the voxels located at the boundaries between white matter fiber tracts and neighboring structures are typical examples of areas affected by the partial volume effect. ${ }^{26,27}$ In the present study the proximal ROI was positioned $4 \mathrm{~mm}$ posterior to the ocular bulb to possibly exclude from the ROI analysis the voxels located at the boundary between white matter tracts and the ocular bulb. Care was also taken to exclude from the analysis ROIs which contained one or more "empty" voxels, and DT images that revealed a contamination of the anterior segment by the posterior aspect of the globe.

In the present study the authors used an anisotropic voxel in diffusion tensor estimation. Oouchi et al. ${ }^{28}$ investigated the effect of voxel size/shape on measured FA and MD by using isotropic $\left(2 \times 2 \times 2 \mathrm{~mm}^{3}\right)$ and nonisotropic voxels $(2 \times 2 \times 6$ $\mathrm{mm}^{3}$ ) selecting a ROI on the transaxial view in different regions of the brain. The authors reported that no differences could be observed between the isotropic and the anisotropic voxel in areas of the brain where no crossing fibers were present, independently of fiber direction. ${ }^{28}$ Thus, the DTI data obtained in the present study using an anisotropic voxel could be considered reliable in relation to the fact that no crossing fibers are present in the optic nerve.

In conclusion, this study's in vivo results indicate that intraorbital optic nerve degeneration is a complex biological process that affects differently the proximal and the distal segments of the intraorbital optic nerve. The present data suggest that DT-MRI parameters may help to provide a better understanding of the pathological interactions that occur in glaucoma. Also, the complementary high sensitivity of FA values with the high specificity of MD values, measured at the proximal site, may provide reliable indexes for the identification of glaucomatous patients at early stages.

\section{References}

1. Ray K, Mookherjee SJ. Molecular complexity of primary open angle glaucoma: current concepts. J Genet. 2009;88:451-467.

2. Cedrone C, Mancino R, Cerulli A, Cesareo M, Nucci C. Epidemiology of primary glaucoma: prevalence, incidence, and blinding effects. Prog Brain Res. 2008;173:3-14.

3. Nickells RW. From ocular hypertension to ganglion cell death: a theoretical sequence of events leading to glaucoma. Can J Ophthalmol. 2007;42:278-287.

4. Quigley HA. New paradigms in the mechanisms and management of glaucoma. Eye (Lond). 2005;19:1241-1248.

5. Morrison JC, Dorman-Pease ME, Dunkelberger GR, Quigley HA. Optic nerve head extracellular matrix in primary optic atrophy and experimental glaucoma. Arch Ophthalmol. 1990; 108:1020-1024.

6. Pena JD, Netland PA, Vidal I, Dorr DA, Rasky A, Hernandez MR. Elastosis of the lamina cribrosa in glaucomatous optic neuropathy. Exp Eye Res. 1998;67:517-524.

7. Furuyoshi N, Furuyoshi M, May CA, Hayreh SS, Alm A, LütjenDrecoll E. Vascular and glial changes in the retrolaminar optic nerve in glaucomatous monkey eyes. Ophthalmologica. 2000; 214:24-32.

8. Hernandez MR. Ultrastructural immunocytochemical analysis of elastin in the human lamina cribrosa. Changes in elastic fibers in primary open-angle glaucoma. Invest Ophthalmol Vis Sci. 1992;33:2891-2903.

9. Quigley HA, Brown A, Dorman-Pease ME. Alterations in elastin of the optic nerve head in human and experimental glaucoma. Br J Ophthalmol. 1991;75:552-557.

10. Quigley HA, Dorman-Pease ME, Brown AE. Quantitative study of collagen and elastin of the optic nerve head and sclera in human and experimental monkey glaucoma. Curr Eye Res. 1991;10:877-888.

11. Crawford DJ, Roberts MD, Sigal IA. Glaucomatous cupping of the lamina cribrosa: a review of the evidence for active progressive remodeling as a mechanism. Exp Eye Res. 2011; 93:133-140.

12. Agoumi Y, Sharpe GP, Hutchison DM, Nicolela MT, Artes PH, Chauhan BC. Laminar and prelaminar tissue displacement during intraocular pressure elevation in glaucoma patients and healthy controls. Ophthalmology. 2011;118:52-59.

13. Roberts MD, Sigal IA, Liang Y, Burgoyne CF, Downs JC. Changes in the biomechanical response of the optic nerve head in early experimental glaucoma. Invest Ophthalmol Vis Sci. 2010;51:5675-5684.

14. Johnson EC, Jia L, Cepurna WO, Doser TA, Morrison JC. Global changes in optic nerve head gene expression after exposure to elevated intraocular pressure in a rat glaucoma model. Invest Ophthalmol Vis Sci. 2007;48:3161-3177.

15. Garaci FG, Bolacchi F, Cerulli A, et al. Optic nerve and optic radiation neurodegeneration in patients with glaucoma: in vivo analysis with 3-T diffusion-tensor MR imaging. Radiology. 2009;252:496-501.

16. Xu J, Sun SW, Naismith RT, Snyder AZ, Cross AH, Song SK. Assessing optic nerve pathology with diffusion MRI: from mouse to human. NMR Biomed. 2008;21:928-940.

17. Hui ES, Fu QL, So KF, Wu EX. Diffusion tensor MR study of optic nerve degeneration in glaucoma. Conf Proc IEEE Eng Med Biol Soc. 2007;2007:4312-4315.

18. Basser PJ, Pierpaoli C. Microstructural and physiological features of tissues elucidated by quantitative-diffusion-tensor MRI. J Magn Reson B. 1996;111:209-219.

19. Pierpaoli C, Jezzard P, Basser PJ, Barnett A, Di Chiro G. Diffusion tensor MR imaging of the human brain. Radiology. 1996;201:637-648.

20. Hodapp E, Parrish RK II, Anderson DR. Clinical Decisions in Glaucoma. St. Louis, MO: The C.V. Mosby Comp; 1993:52-61.

21. Anderson DR. Ultrastructure of human and monkey lamina cribrosa and optic nerve head. Arch Ophthalmol. 1969;82: 800-814.

22. Pierpaoli C, Barnett A, Pajevic S, et al. Water diffusion changes in Wallerian degeneration and their dependence on white matter architecture. Neuroimage. 2001;13(6, pt 1):11741185.

23. Hasan KM. Diffusion tensor eigenvalues or both mean diffusivity and fractional anisotropy are required in quantitative clinical diffusion tensor MR reports: fractional anisotropy alone is not sufficient. Radiology. 2006;239:611-613.

24. Beaulieu C. The basis of anisotropic water diffusion in the nervous system: a technical review. NMR Biomed. 2002;15: 435-455.

25. Barker GJ. Diffusion-weighted imaging of the spinal cord and optic nerve. J Neurol Sci. 2001;186(suppl 1):S45-S49.

26. Stieltjes B, Kaufmann WE, van Zijl PC, et al. Diffusion tensor imaging and axonal tracking in the human brainstem. Neuroimage. 2001;14:723-735.

27. Alexander AL, Hasan KM, Lazar M, Tsuruda JS, Parker DL. Analysis of partial volume effects in diffusion-tensor MRI. Magn Reson Med. 2001;45:770-780.

28. Oouchi H, Yamada K, Sakai K, et al. Diffusion anisotropy measurement of brain white matter is affected by voxel size: underestimation occurs in areas with crossing fibers. $A J N R$ Am J Neuroradiol. 2007;28:1102-1106. 